ARTICLE

\title{
A common limiter circuit for opioid choice and relapse identified in a rodent addiction model
}

\author{
Jasper A. Heinsbroek (10 1, Giuseppe Giannotti (1) 1,2, Mitchel R. Mandel1,2, Megan Josey 1,2, Gary Aston-Jones ${ }^{3}$,
} Morgan H. James ${ }^{3,4} \&$ Jamie Peters (iD) ${ }^{1,2}$ 瓜

Activity in numerous brain regions drives heroin seeking, but no circuits that limit heroin seeking have been identified. Furthermore, the neural circuits controlling opioid choice are unknown. In this study, we examined the role of the infralimbic cortex (IL) to nucleus accumbens shell (NAshell) pathway during heroin choice and relapse. This model yielded subpopulations of heroin versus food preferring rats during choice, and choice was unrelated to subsequent relapse rates to heroin versus food cues, suggesting that choice and relapse are distinct behavioral constructs. Supporting this, inactivation of the IL with muscimol produced differential effects on opioid choice versus relapse. A pathway-specific chemogenetic approach revealed, however, that the IL-NAshell pathway acts as a common limiter of opioid choice and relapse. Furthermore, dendritic spines in IL-NAshell neurons encode distinct aspects of heroin versus food reinforcement. Thus, opioid choice and relapse share a common addiction-limiting circuit in the IL-NAshell pathway.

\footnotetext{
${ }^{1}$ Department of Anesthesiology, University of Colorado Anschutz Medical Campus, Aurora, CO, USA. ${ }^{2}$ Department of Pharmacology, University of Colorado Anschutz Medical Campus, Aurora, CO, USA. ${ }^{3}$ Brain Health Institute, Rutgers University and Rutgers Biomedical and Health Sciences, Piscataway, NJ, USA.

${ }^{4}$ Department of Psychiatry, Robert Wood Johnson Medical School, Rutgers University, Piscataway, NJ, USA. 凶email: jamie.l.peters@cuanschutz.edu
} 
O pioid addiction is a major public health threat ${ }^{1}$ and understanding the neural basis of opioid choice and relapse is essential to the development of novel treatment interventions. From rodent preclinical models, some components of the opioid relapse circuitry have been established, including projections from the prefrontal cortex to the nucleus accumbens, a proposed final common pathway to relapse. This projection is functionally segregated into a dorsal and a ventral circuit ${ }^{2,3}$. Whereas the dorsal component from the prelimbic cortex to nucleus accumbens core (NAcore) drives relapse for multiple drugs of abuse across multiple models ${ }^{4}$, the function of the ventral projection from infralimbic (IL) cortex to nucleus accumbens shell (NAshell) is less clear. While pharmacological manipulations of the IL and the NAshell suggest a driver function for these individual brain regions in heroin relapse $\mathrm{e}^{5-7}$, a pathwayspecific approach for this circuit has not been explicitly utilized in animal models of opioid seeking. Notably, such an approach has revealed a limiter function for the $\mathrm{IL} \rightarrow \mathrm{NAshell}$ pathway for both cocaine and alcohol seeking ${ }^{8-12}$

By comparison to our knowledge of the neural circuitry of relapse, little is known about the neural circuitry underlying the choice between drug versus natural reward. This is striking given that clinical studies have shown nondrug rewards can prolong abstinence from drug use in humans ${ }^{13}$. Preclinical studies support this in that rodents typically prefer natural rewards over drug rewards under conditions of mutually exclusive choice ${ }^{14-19}$. This behavior has been called voluntary abstinence because animals completely refrain from using drugs in the presence of a natural reward option, supporting the use of natural reinforcers in contingency management therapy ${ }^{20}$. While the neural circuitry controlling relapse after voluntary abstinence is beginning to be mapped $^{21,22}$, we know virtually nothing about the neural basis of opioid choice. Importantly, opioid use disorder in humans is characterized by a substantial population of drug users that become dependent on the drug 23,24 and choose opioids over natural rewards. Thus, we wanted to employ a model wherein individual differences in opioid choice would yield a population average of $\sim 50 \%$ choice between drug versus natural reward, allowing us to shift choice behavior with functional brain-site manipulations (e.g., avoiding a floor/ceiling effect in choice behavior).

Here we investigated the role of the IL $\rightarrow$ NAshell pathway in both opioid choice and relapse in a rat self-administration model that identifies a substantial population of rats that prefer/ choose heroin over food reward and results in a population average of $\sim 50 \%$ choice for heroin over food. In order to understand whether heroin or food demand predicts choice or relapse behavior, we applied principles from the field of behavioral economics. Relationships between $\mathrm{Q}_{0}$ (theoretical consumption when the price is free), alpha ( $\alpha$, demand elasticity), Pmax (maximum price paid before consumption declines), EV (essential value of each reward), and choice and relapse behavior indicated that choice and relapse are conceptually distinct constructs. Consistent with this, pharmacological inactivation of the IL cortex increased heroin choice, but decreased heroin relapse, while having no effect on food relapse. However, targeting the IL cortex outputs to the NAshell specifically using a pathway-specific chemogenetics strategy revealed that this pathway is necessary for limiting heroin choice and heroin relapse, but not food relapse. Furthermore, measures of dendritic spine morphology and density in IL $\rightarrow$ NAshell neurons differentially correlate with specific variables of heroin versus food motivation. The data support a common limiter function for the IL $\rightarrow$ NAshell pathway in heroin seeking under both choice and relapse conditions.

\section{Results}

Heroin is a stronger reinforcer than food. Representative demand curves for heroin and food self-administration over increasing price points from a single animal are shown in Fig. 1a, b. Comparing between food and heroin demand for each rat revealed that motivation for heroin is significantly higher as indicated by higher Pmax (Fig. 1c; paired $t$-test: $t_{(26)}=6.34, p=$ $5.12 \times 10^{-7}$ ) and normalized nPmax (Supplementary Fig. 1c; paired $t$-test: $\left.t_{(26)}=4.60, p=4.78 \times 10^{-5}\right)$ and Omax values (Supplementary Fig. 1d; paired $t$-test: $t_{(26)}=7.64, p=2.10 \times 10^{-8}$ ). In addition, demand was less elastic for heroin than food as indicated by lower $\alpha$ values (Fig. 1d; paired $t$-test: $t_{(26)}=11.2, p=$ $9.70 \times 10^{-12}$ ). Moreover, the unit-independent $\mathrm{EV}$ which allows for direct comparisons between different reinforcers ${ }^{25}$ was significantly higher for heroin (Fig. 1e; paired $t$-test: $t_{(26)}=7.54, p=$ $\left.2.61 \times 10^{-8}\right)$. Finally, consumption at low prices $\left(\mathrm{Q}_{0}\right)$ for both heroin and food indicated that rats respond significantly more for food than for heroin under these conditions (Fig. 1f; paired $t$-test: $\left.t_{(26)}=4.87, p=2.40 \times 10^{-5}\right)$. Altogether, these results indicate that rats have a higher motivation for heroin and that heroin is considered more essential at higher price points than food reward in this model.

Heroin choice and relapse are distinct behavioral constructs. Following the behavioral economic phase, choice procedures commenced, and rats had to make mutually exclusive choices between heroin versus food. After choice stabilized, a range of choice behavior emerged, with a population average of $\sim 50 \%$ heroin choice. This model is ideal to examine the neural basis of choice between heroin and natural reward. To examine the relationship between choice and other behavioral measures, rats were arbitrarily subdivided into Heroin preferers $(>60 \%$ heroin choice) versus Food preferers $(<40 \%$ heroin choice) groups (Fig. 2a). Following choice testing and reacquisition of food and heroin self-administration, rats underwent cued relapse tests with each lever presented independently, as during training. No differences were observed in response rates for food versus heroin cues, and relapse did not correlate with heroin choice (Fig. 2b).

We applied a principal component analysis to assess the relationships between behavioral economic variables, total intake of food or heroin, heroin choice, and relapse. Behavioral economic variables, total intake, and relapse clustered along with the first component and separated by reinforcer type (food versus heroin) along with the second component (with a notable exception for heroin $\mathrm{Q}_{0}$, which clustered with food variables). By contrast, heroin choice separated along with the first component from all other variables, suggesting that heroin choice is distinct from demand, intake, and relapse (Fig. 2c). We propose that choice may represent a cognitive measure of addiction, whereas the other variables may reflect motivational components. Consistent with prior studies ${ }^{26-28}$, heroin relapse (Fig. $2 \mathrm{~d}$ ), but not food relapse (Fig. 2e), negatively correlated with the reinforcer $(\log )$ a value, indicating that heroin relapse rates are higher in rats with more inelastic demand. Total intake during self-administration also correlated with behavioral economic variables by reinforcer type (see Supplementary Fig. 2 for a complete correlation matrix), suggesting that motivation for each reinforcer may increase as consumption history increases ${ }^{29}$, or alternatively, that higher motivational drive leads to increased consumption. Corroborating the correlational analysis, statistical comparisons between food preferers and heroin preferers did not reveal any differences between these groups in behavioral economics variables or relapse (Supplementary Fig. 3), with the exception that food preferring rats had elevated $Q_{0}$ compared to heroin preferers (Supplementary Fig. 3e). 
a

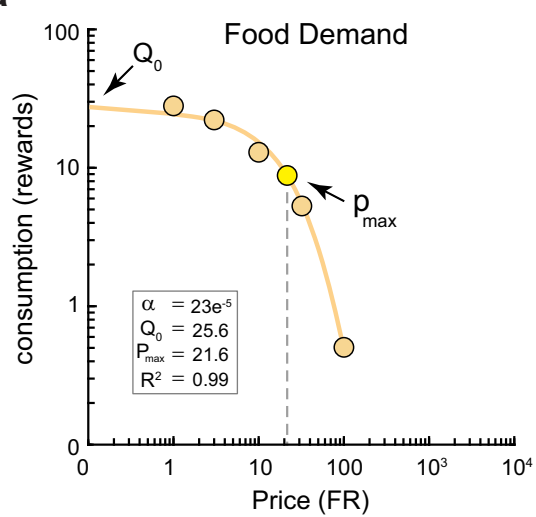

b

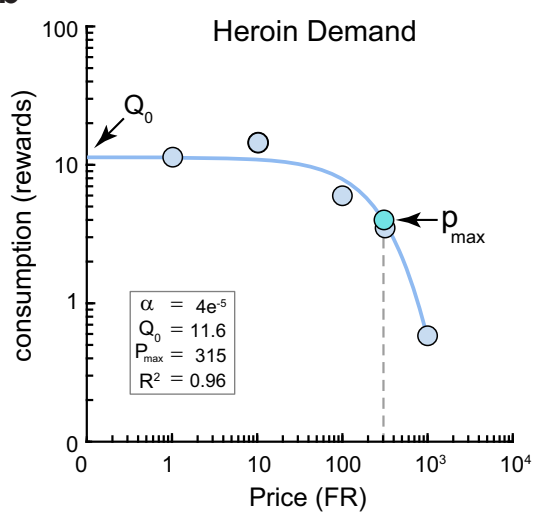

f
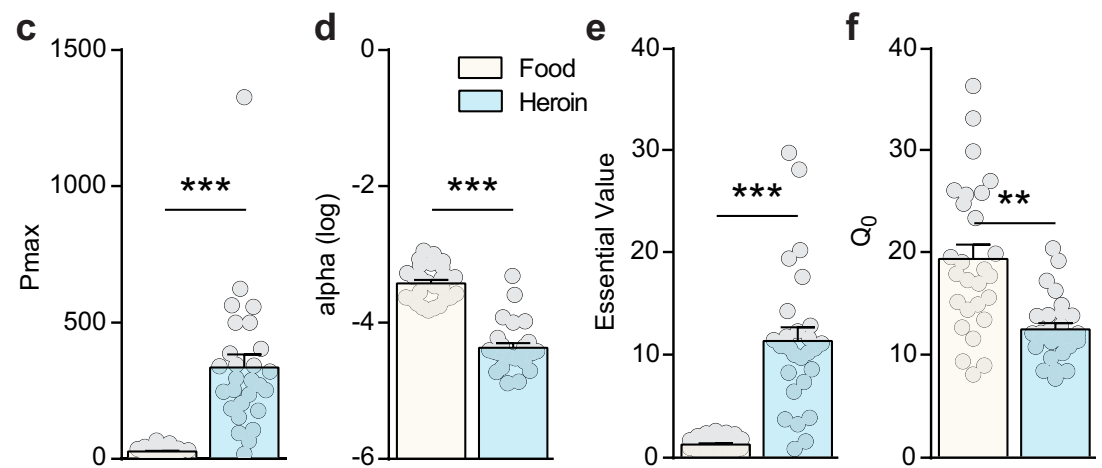

Fig. 1 Rats value heroin more than food. Representative demand curves for food (a) and heroin (b) for a single rat. c Rats are more motivated for heroin than for food as assessed by a higher Pmax, the maximum price animals are willing to pay to maintain consumption before levels drop off (two-tailed paired $t$-test, $t_{(26)}=$ $6.34, p=5.12 \times 10^{-7}$ ). $\mathbf{d}$ Heroin demand is less elastic than food demand (responding drops more rapidly for food as price increases; two-tailed paired $t$-test, $t_{(26)}$ $=11.2, p=9.70 \times 10^{-12}$ ) and correspondingly e heroin has a higher essential value than food (two-tailed paired $t$-test, $\left.t_{(26)}=7.54, p=2.61 \times 10^{-8}\right)$. $\mathbf{f}$ Rats consume more food than heroin at low price points as indicated by a higher $Q_{0}$ value (two-tailed paired $t$-test, $t_{(26)}=4.87, p=2.40 \times 10^{-5}$ ). $n=27$ rats. ${ }^{\star \star \star} p<$ $0.001,{ }^{\star \star} p<0.01$ comparing between heroin and food behavioral economic variables. Data were presented as mean \pm SEM (c-f).

Silencing IL neurons differentially alters heroin choice and relapse. A separate group of rats was implanted with chronic indwelling cannulae aimed at IL and infused with the GABA-A receptor agonist muscimol (IL-MUS, Fig. 3a) or vehicle (IL-PBS) prior to the choice or relapse tests. This group did not have the extensive training history that the behavioral economics group had, yet opioid choice again stabilized at $~ 50 \%$ (Fig. 3b). Due to the relatively short-lasting effects of intracranial MUS ${ }^{30}$, analyses were restricted to the first hour of the $(3 \mathrm{~h})$ choice sessions on test days. IL-MUS significantly shifted choice toward heroin (Fig. 3c; paired $t$-test: $t_{(10)}=2.39, p=0.038$ ). Following choice testing and reacquisition of food and heroin self-administration, rats underwent cued relapse tests with both levers present simultaneously, as during training. Relapse rates for heroin cues were significantly higher than those for food cues (Fig. 3d; two-way RM-ANOVA main effect of lever: $\left.F_{(1,13)}=11.70, p=0.005\right)$. However, once again, there were no differences in relapse rates between heroin versus food preferers (Supplementary Fig. 4), reinforcing the notion that choice and relapse are distinct behavioral constructs. Interestingly, IL-MUS reduced heroin relapse (Fig. 3d; Bonferroni post hoc $p=0.038$ ), but not food relapse. Thus, endogenous activity in IL cortex limits opioid choice but drives relapse.

The IL $\rightarrow$ NAshell pathway limits both heroin choice and relapse. We used a combinatorial virus approach to restrict the expression of designer receptors activated exclusively by designer drugs
(DREADDs) to the $\mathrm{IL} \rightarrow$ NAshell pathway ${ }^{31,32}$. The efficacy of the DREADD ligand J60 to reduce neuronal activity in animals expressing the inhibitory Gi-DREADD (Fig. 4a) was verified in an independent cohort of rats. J60 significantly reduced IL Fos expression induced by a priming injection of cocaine in DREADD-infected neurons (Fig. $4 \mathrm{~b}, \mathrm{c}$; unpaired $t$-test: $t_{(13)}=$ 2.23, $p=0.044)$. Chemogenetic inhibition of the $\mathrm{IL} \rightarrow$ NAshell pathway shifted choice toward heroin (Fig. 4d; paired $t$-test: $t_{(6)}=3.19, p=0.018$ ) and increased heroin relapse (Fig. 4e; paired $t$-test: $t_{(6)}=2.73, p=0.034$ ), without altering food relapse (Fig. 4f).

The efficacy of J60 to induce neuronal activity in animals expressing the excitatory Gq-DREADD (Fig. 5a) was verified in an independent cohort of rats. J60 significantly induced IL Fosexpression in DREADD-infected neurons (Fig. 5b, c; unpaired $t$ test: $\left.t_{(8)}=3.51, p=0.008\right)$. However, chemogenetic activation of the IL $\rightarrow$ NAshell pathway using the excitatory Gq-DREADD did not alter heroin choice (Fig. 5d), nor did it impact heroin relapse (Fig. 5e) or food relapse (Fig. 5f). These data indicate that activity in the IL $\rightarrow$ NAshell pathway is necessary (Fig. 4), but not sufficient (Fig. 5), to limit heroin choice and heroin relapse. When we employed a standard self-administration, extinction, and cued reinstatement model ${ }^{9}$, chemogenetic activation of the $\mathrm{IL} \rightarrow$ NAshell pathway was sufficient to reduce heroin relapse (Supplementary Fig. 5). Thus, under some conditions, it may be possible to exploit the limiter function of the $\mathrm{IL} \rightarrow$ NAshell pathway to reduce relapse. 

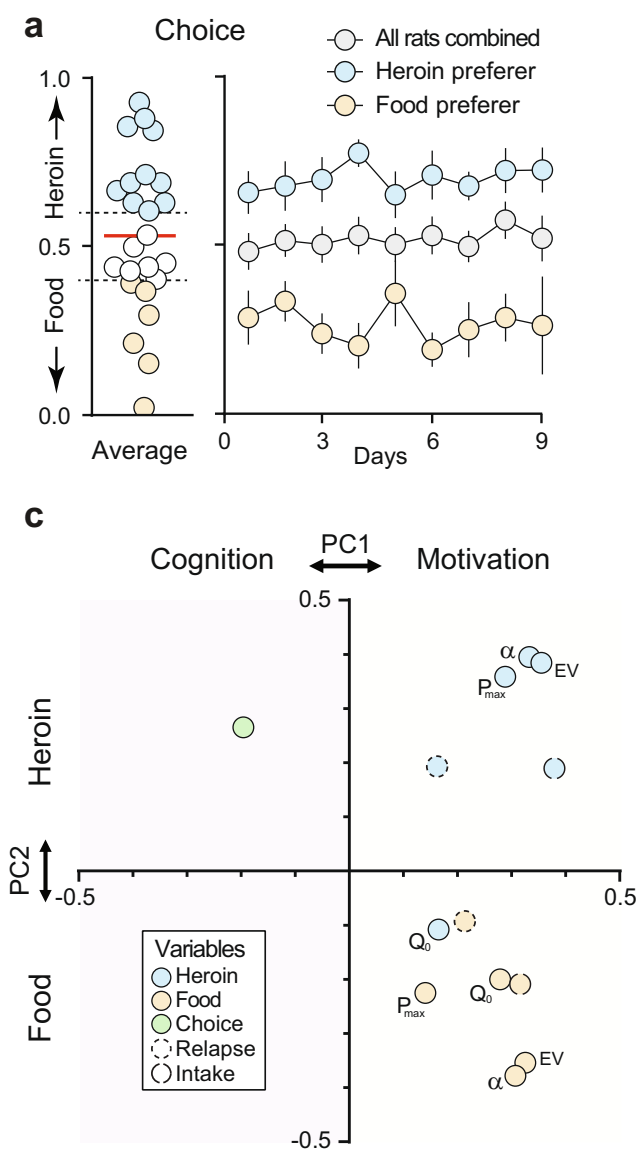

b
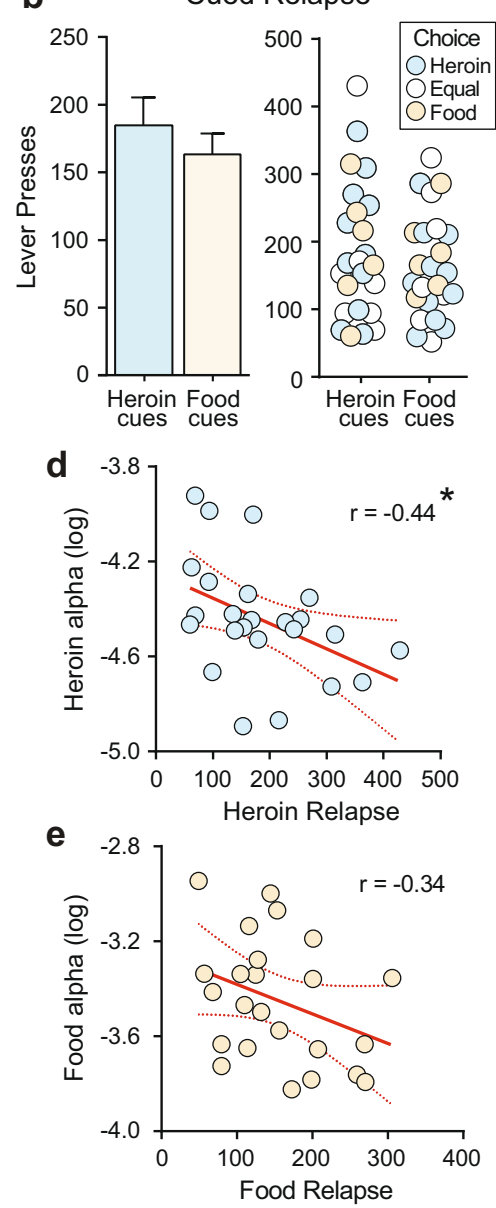

Fig. 2 Heroin choice and relapse are distinct behaviors. a Overall rats choose food and heroin equally, with a subset of rats preferring heroin ( $>60 \%$ heroin choice, $n=11$ rats) versus food ( $>60 \%$ food choice, $n=6$ rats). b Rats reinstate food and heroin cues equally, and relapse does not differ between food and heroin preferring animals. $\mathbf{c}$ Heroin relapse clusters with heroin variables and food relapse with food variables (and heroin $Q_{0}$ ), but the choice is distinct from these variables in principal component space. Blue colors indicate heroin variables, orange food variables, and choice is labeled green. $\mathbf{d}$ Heroin $\alpha$ negatively correlates with heroin relapse (Pearson's $r=-0.44, p=0.03$ ). e Food $\alpha$ does not correlate with food relapse $n=24$ rats $(\mathbf{a}-\mathbf{e})$. ${ }^{\star} p<$ 0.05 significant correlation. Data were presented as mean \pm SEM $(\mathbf{a}, \mathbf{b})$.

IL $\rightarrow$ NAshell spines encode heroin choice and relapse. At the end of the experiment, structural analyses were performed on dendritic segments from IL $\rightarrow$ NAshell neurons (using the mCherry tag on the DREADD, Fig. 6a). Spine density (Fig. 6b) and morphology (Fig. 6f) were measured as the number of spines per dendritic segment or the average spine head diameter per dendritic segment, respectively. This analysis revealed bidirectional relationships between these structural measures and heroin versus food reinforcement. IL $\rightarrow$ NAshell neuron spine density positively correlated with food relapse (Fig. $6 c ; r=0.68, p=$ 0.043 ) and negatively correlated with food (log) a value (Fig. $6 \mathrm{~d} ; r$ $=-0.67, p=0.047)$. Further analysis of the cumulative frequency distribution of inter-spine intervals (the space between individual spines) revealed a difference between heroin versus food preferers, with food preferers having significantly smaller inter-spine intervals (Fig. 6e; KS2 $=0.07, p=1.29 \times 10^{-5}$ ). By contrast, spine morphology correlated with measures of heroin, but not food, motivation. Spine head diameter positively correlated with heroin relapse (Fig. 6g; $r=0.71, p=0.030$ ) and negatively correlated with heroin $(\log )$ a value (Fig. $6 \mathrm{~h} ; r=-0.68, p=0.043$ ). No differences were observed in the cumulative frequency distribution of spine head diameter between heroin versus food prefers (Fig. 6i), and no other significant correlations were observed besides those reported above.

\section{Discussion}

These results identify the first neural circuit that acts as an endogenous limiter of heroin seeking, namely the IL $\rightarrow$ NAshell pathway. These results are congruent with findings indicating that this pathway is recruited by extinction learning to limit cocaine seeking $8,9,33$, and suggest that giving animals a choice between drug versus natural rewards, which has been likened to a form of contingency management therapy ${ }^{18}$, may recruit similar circuits as extinction training, another form of cognitive behavioral therapy. Furthermore, these results identify the first neural circuit implicated in opioid choice; specifically the IL $\rightarrow$ NAshell pathway similarly functions as a limiter of heroin choice (when food is an alternative reward). Correlational and principal components analyses support the notion that heroin choice and relapse are distinct constructs, making it all the more striking that the limiter function of the IL $\rightarrow$ NAshell pathway extends to both forms of addictive behavior. Nonetheless, other IL outputs may mediate heroin relapse, as global inactivation of the IL cortex did not elicit parallel effects on relapse, as it did on choice.

Behavioral economics has been applied to the study of human substance abuse and was shown, along with choice-based relapse, to relate to measures of dependence and abuse ${ }^{34}$. It has further been suggested that choice (between drug and alternative reward) may reveal human subpopulations that could benefit from 

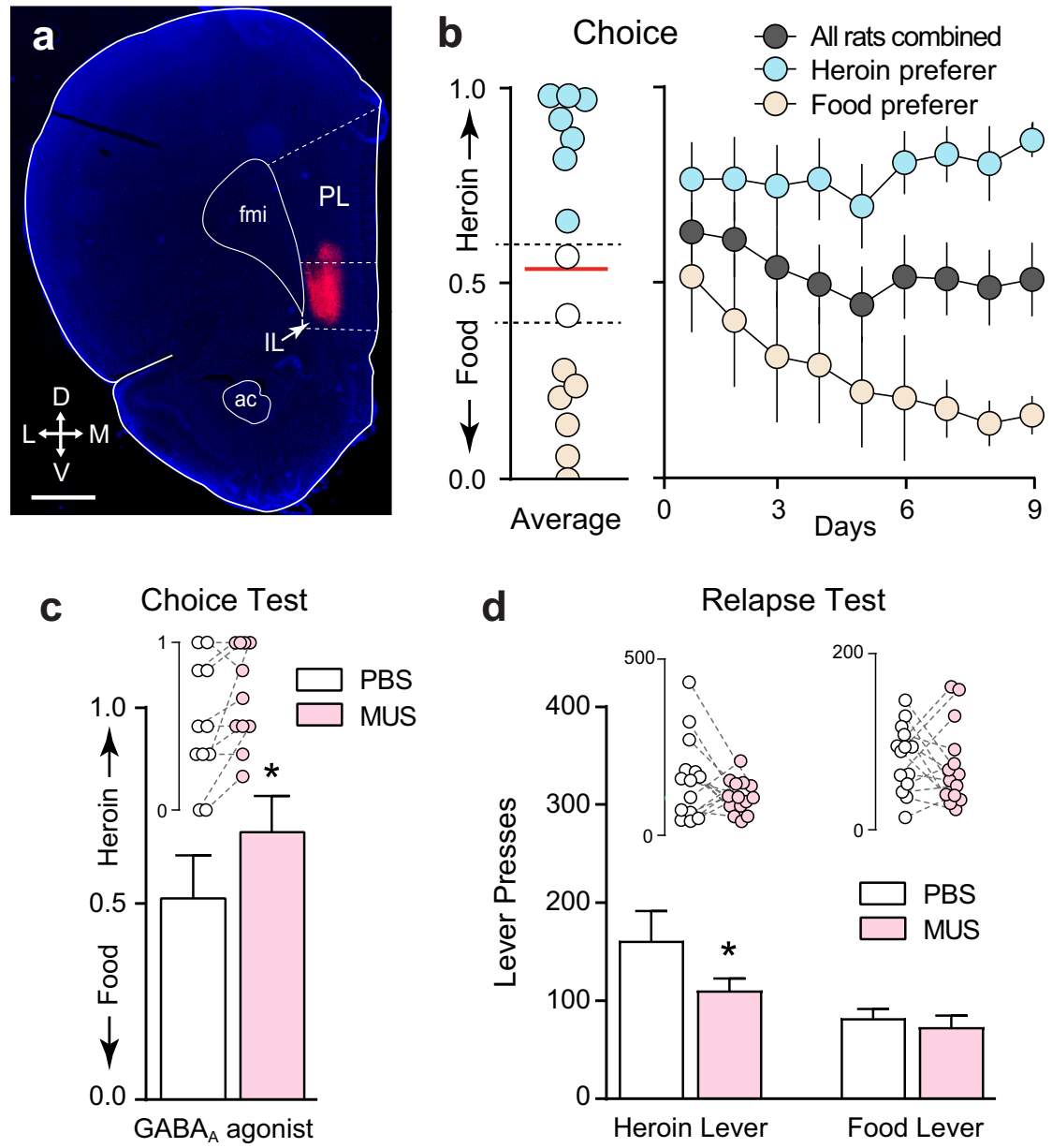

Fig. 3 Silencing IL neurons differentially alters heroin choice and relapse. a Representative micrograph showing cannula placement and infusion of the fluorescent $\mathrm{GABA}_{\mathrm{A}}$ receptor agonist muscimol (MUS) into IL (scale bar $=1000 \mu \mathrm{m}$ ). PL prelimbic cortex, fmi forceps minor of the corpus callosum, ac anterior commissure, arrows indicate anatomical orientation along with dorsoventral (DV) and mediolateral (ML) axes. b Overall rats choose heroin and food equally, but distinct populations of rats become heroin preferring $(n=7)$ or food preferring $(n=6)$ over time. c Muscimol microinjection into IL potentiates heroin preference during choice (two-tailed paired $t$-test, $t_{(10)}=2.39, p=0.04 ; n=11$ rats). $\mathbf{d}$ Muscimol microinjection reduced cued relapse during a test in which both food and heroin levers and cues were present but rewards were unavailable (two-way ANOVA $F_{(1,13)}=11.70, p=4.56 \times 10^{-3}$, Bonferroni post hoc test, $p=0.04 ; n=14$ rats). ${ }^{\star} p<0.05$ comparing between vehicle and MUS. Data represented as mean \pm SEM.

contingency management therapy ${ }^{35-37}$. Corroborating this, preclinical studies consistently show a negative relationship between $\alpha$ and relapse rates ${ }^{27,38}$, consistent with our findings here for heroin. However, principal component and correlational analyses of behavioral economic variables, choice, and relapse clearly identified choice as a distinct behavioral construct. Whereas behavioral economic variables associated with each other, predominantly clustering by reward type (heroin versus food), they also clustered with relapse and total intake (consumption history). Thus, these variables likely reflect a larger behavioral construct, possibly motivation. By contrast, the opioid choice fell within its own principal components space, which we propose represents the construct of cognition. While purely theoretical, regardless of the hypothetical construct assigned to principal component space in these analyses, heroin choice clearly separated from the other behavioral variables, including heroin relapse.

Since global inactivation of IL cortex with IL-MUS produced a similar upward shift in heroin choice as IL $\rightarrow$ NAshell pathwayspecific inhibition, the opioid choice may predominantly recruit this IL output (NAshell). Similarly, IL $\rightarrow$ NAshell pathway inhibition produced an increase in heroin, but not food, relapse. Thus, the $\mathrm{IL} \rightarrow$ NAshell functions as a common limiter of these distinct behaviors and may reflect the top-down cognitive control IL exerts over NAshell-motivational processes ${ }^{39,40}$. Though $\mathrm{IL} \rightarrow$ NAshell activation was not sufficient to limit opioid choice or relapse during acute withdrawal after choice procedures, it was capable of reducing cued heroin relapse in a standard extinctionreinstatement model. It is possible that $\mathrm{IL} \rightarrow$ NAshell activity is already at a ceiling after choice procedures and/or acute withdrawal, but may be further enhanced to limit relapse after extinction and/or protracted withdrawal. Interestingly, global ILMUS inactivation actually decreased heroin, but not food, relapse, an effect opposite that of IL $\rightarrow$ NAshell pathway-specific inhibition. This suggests that the IL cortex can also function as a driver of heroin seeking, consistent with other reports ${ }^{5-7}$. Nonetheless, our findings in this heroin model are consistent with observations that IL neuronal ensembles exert inhibitory control over alcohol seeking $^{41,42}$. As specific IL outputs were not identified in the latter studies, these apparent discrepancies may relate to different IL outputs being engaged under different conditions.

Surprisingly, very little is known about the neural circuits controlling the choice between opioid versus natural reward. However, choice behavior likely involves distinct prefrontal circuits that attribute and compare the expected value of different rewards, and that monitor behavioral strategies. Within the IL 

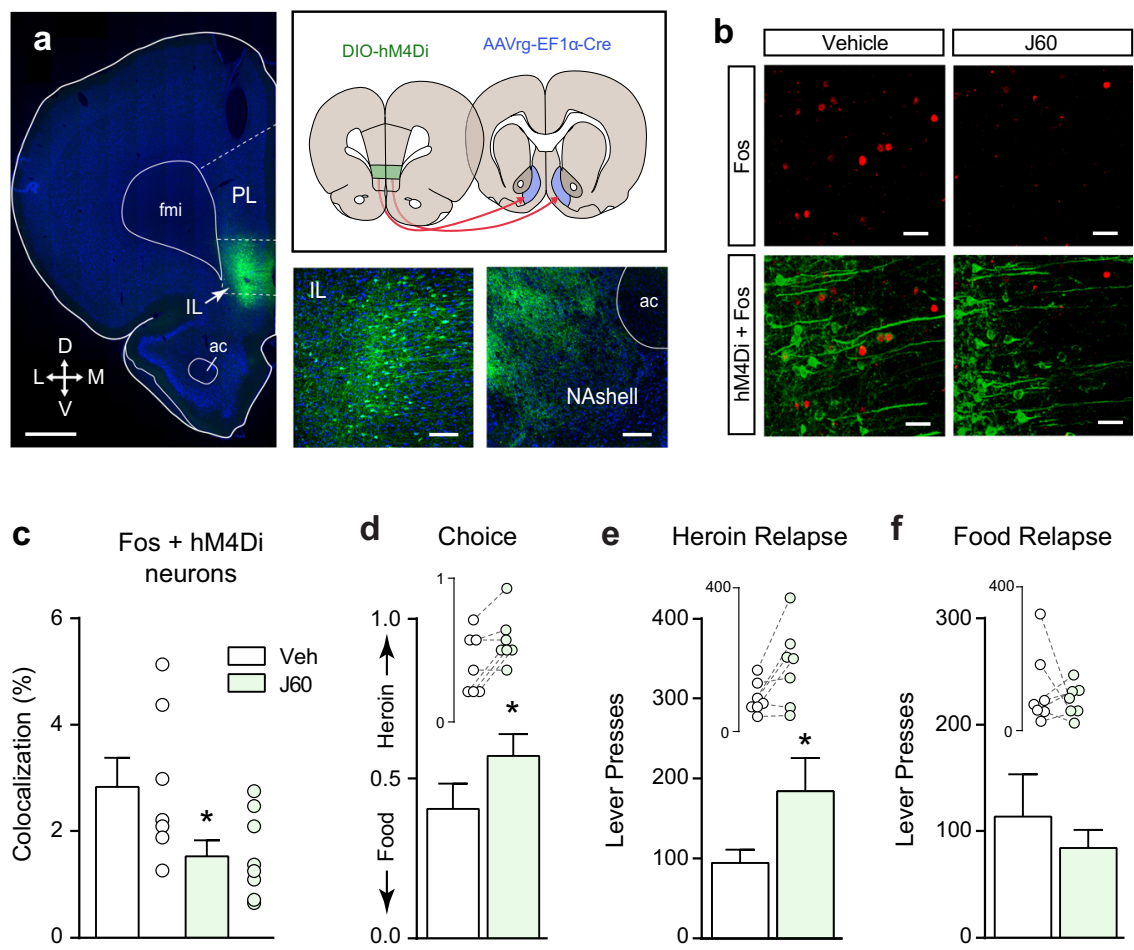

Fig. 4 Inhibiting neural activity in the IL $\rightarrow$ NAshell pathway increases heroin choice and relapse. a Representative micrograph showing Gi-DREADD ( $\mathrm{hM} 4 \mathrm{Di}$ ) expression in the IL $\rightarrow$ NAshell pathway and the spread of virus injections in both IL and NAshell (scale bar $=1000 \mu \mathrm{m}$ ). Only rats with virus expression confined to IL were included in the study $(n=7)$. Inserts show high magnification (20x) DREADD expression in the IL (left; scale bar $=100 \mu \mathrm{m}$ ) and (10x) labeling of IL axons in the NAshell (right; scale bar $=200 \mu \mathrm{m}$ ). PL prelimbic cortex, fmi forceps minor of the corpus callosum, ac anterior commissure, arrows indicate anatomical orientation along with dorsoventral (DV) and mediolateral (ML) axes. $\mathbf{b}$, c Chemogenetic inhibition of IL $\rightarrow$ NAshell neurons with the DREADD agonist $J 60$ reduces cocaine-induced Fos expression in virus expressing neurons (two-tailed unpaired $t$-test, $t_{(13)}=2.23, p=$ 0.04; vehicle: $n=7, J 60: n=8$ rats; scale bar $(\mathbf{b})=30 \mu \mathrm{m}$ ). $\mathbf{d}$ Inhibition of the IL $\rightarrow$ NAshell pathway shifts choice towards heroin (two-tailed paired $t$-test, $\left.t_{(6)}=3.19, p=0.02\right)$. e Inhibition of the IL $\rightarrow$ NAshell pathway does not affect food relapse. $\mathbf{f}$ Inhibition of the IL $\rightarrow$ NAshell pathway potentiates heroin relapse (two-tailed paired $t$-test, $t_{(6)}=2.73, p=0.03 . n=7$ rats $(\mathbf{d}-\mathbf{f}) .{ }^{*} p<0.05$ comparing vehicle and J60. Data were represented as mean \pm SEM.

cortex, neuronal ensembles encoding alcohol versus saccharin rewards (two tastant rewards) were found to exhibit some overlap, but an equal portion of these ensembles were distinct ${ }^{43}$. Although the IL cortex has not to our knowledge been examined in the context of opioid choice, within the orbitofrontal cortex, which receives input from the IL cortex ${ }^{44}$, distinct populations of neurons encode food or heroin choice ${ }^{45}$. The adjacent anterior insular cortex and its projection to the central amygdala regulate methamphetamine relapse following voluntary abstinence by food choice ${ }^{22}$, and expression of a $\gamma$-aminobutyric acid (GABA) GABA transporter (GAT-3) in the central amygdala has been linked to alcohol choice in both rodents and humans ${ }^{46}$. Other anterior insular projections to the NAcore mediate choice between two distinct food rewards, and downstream $\mu$ opioid receptors in the NAcore are involved ${ }^{47}$. Mu opioid receptors are also highly expressed in the NAshell, and concentrated in hedonic hot spots which directly mediate opioid and food reward ${ }^{48-50}$. Activation of these $\mu$ opioid receptors reduces neuronal activity and increases food consumption, an effect that is consistent with other pharmacological means of inhibiting NAshell activity ${ }^{51,52}$. This may in part account for observations that prolonged heroin self-administration, which directly activates $\mu$ opioid receptors, increases motivation for both heroin and sweet reward ${ }^{29}$.

Analyses of dendritic spines suggest that specific structural features in IL $\rightarrow$ NAshell neurons may encode distinct aspects of heroin versus food reinforcement, with spine head morphology correlating with heroin measures (heroin relapse and demand elasticity), and spine density with food measures (food relapse and food elasticity). Opioid choice, by contrast, may specifically relate to the inter-spine interval, with lower opioid choice reflected in more closely spaced spines along the dendritic shaft. How these structural markers relate to the physiological activity of these neurons, and the specificity of these markers to the $\mathrm{IL} \rightarrow \mathrm{NAshell}$ pathway remains to be determined. It is nonetheless striking that these post hoc structural analyses distinguished heroin versus food relapse in morphological versus densitometric domains, despite the fact that heroin relapse correlated with food relapse in this study (Supplementary Fig. 2). Notably, however, heroin and food relapse did not correlate in the IL-MUS study (Supplementary Fig. 4). Thus, presenting each lever independently as in the former study may have resulted in greater generalization across levers, even though the order in which they were presented was counterbalanced. This may also explain why heroin and food relapse rates did not differ in the behavioral economics study, but did in the IL-MUS study. When both levers were presented simultaneously as in the latter study, thus forcing the rats to choose how they allocated their seeking behavior, heroin relapse rates were higher than those for food.

In our choice model, we identified subpopulations of heroin preferring versus food preferring rats under equivalent price conditions (FR3). This contrasts with findings from other choice models, which have been dubbed voluntary abstinence models ${ }^{18,53}$ due to the majority of rats exclusively choosing food reward over drug reward. Still, others have been able to produce these subpopulations by increasing the delay to food reward (i.e., discounting food $)^{54}$ or by extending the duration of daily drug self-administration (e.g., using long-access procedures) ${ }^{55-57}$. Our model yields $\sim 50 \%$ heroin choice (without altering conditions 

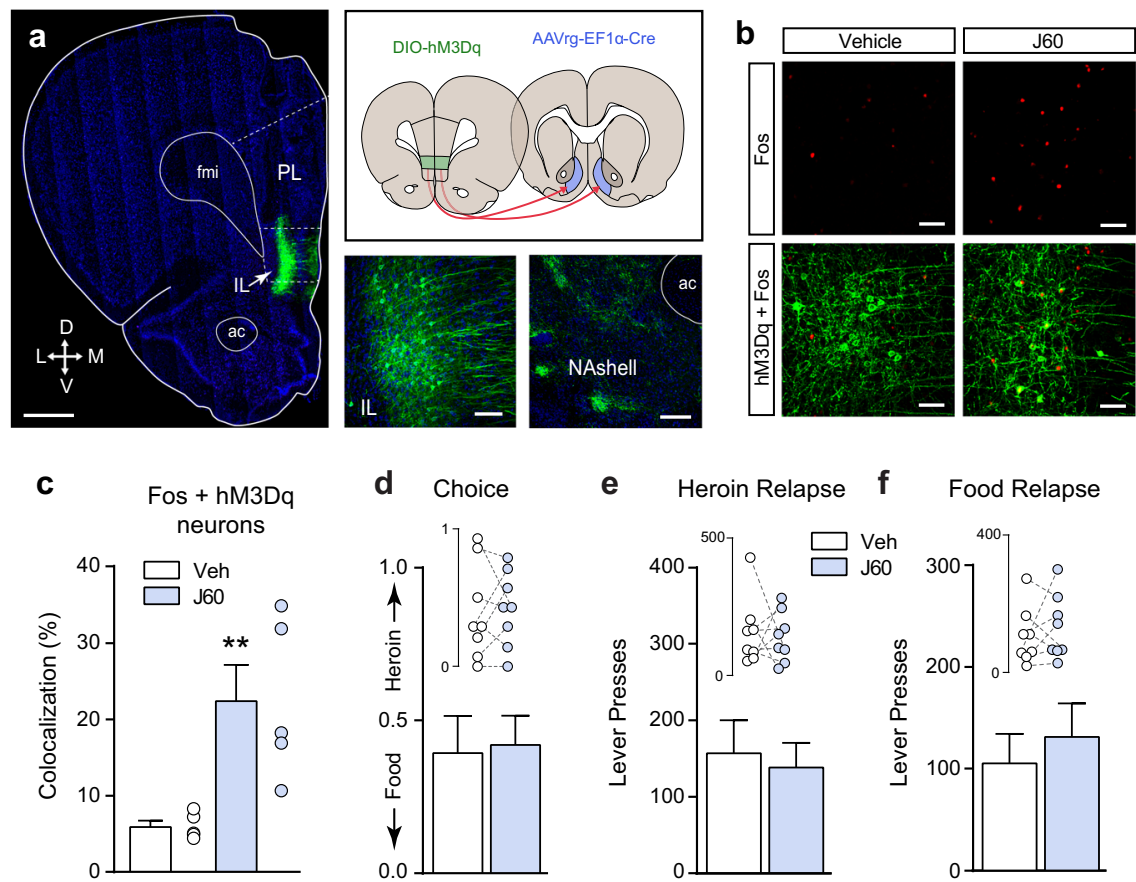

Fig. 5 Stimulating neuronal activity in the IL $\rightarrow$ NAshell pathway does not alter heroin choice or relapse. a Micrograph showing Gq-DREADD (hM3Dq) expression in IL $\rightarrow$ NAshell neurons (scale bar $=1000 \mu \mathrm{m}$ ). Inserts show high magnification $(20 \mathrm{x})$ DREADD expression in the IL (left; scale bar $=100 \mu \mathrm{m}$ ) and (10x) labeling of IL axons in the NAshell (right; scale bar $=200 \mu \mathrm{m})$. Only rats with virus expression confined to IL were included in the study $(n=8)$. PL prelimbic cortex, fmi forceps minor of the corpus callosum, ac anterior commissure, arrows indicate anatomical orientation along with dorsoventral (DV) and mediolateral (ML) axes. b, c Chemogenetic stimulation of IL $\rightarrow$ NAshell neurons with the DREADD agonist J60 induces Fos expression in virus expressing neurons (two-tailed unpaired $t$-test, $t_{(8)}=3.51, p=0.01$; vehicle: $n=5, J 60: n=5$ rats; scale bar $(\mathbf{b})=30 \mu \mathrm{m}$ ). $\mathbf{d}$ Stimulation of the IL $\rightarrow$ NAshell pathway does not affect choice or relapse to food (e) or heroin (f) cues. $n=8$ rats $(\mathbf{d}-\mathbf{f}){ }^{\star \star} p<0.01$ comparing vehicle and J60. Data were represented as mean \pm SEM

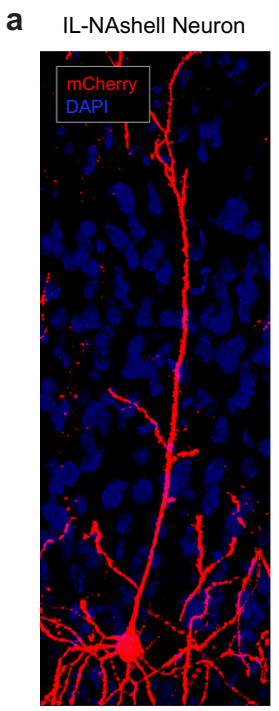

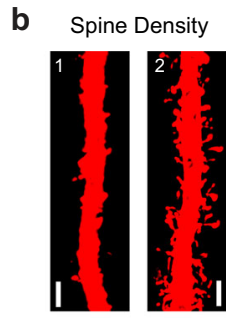
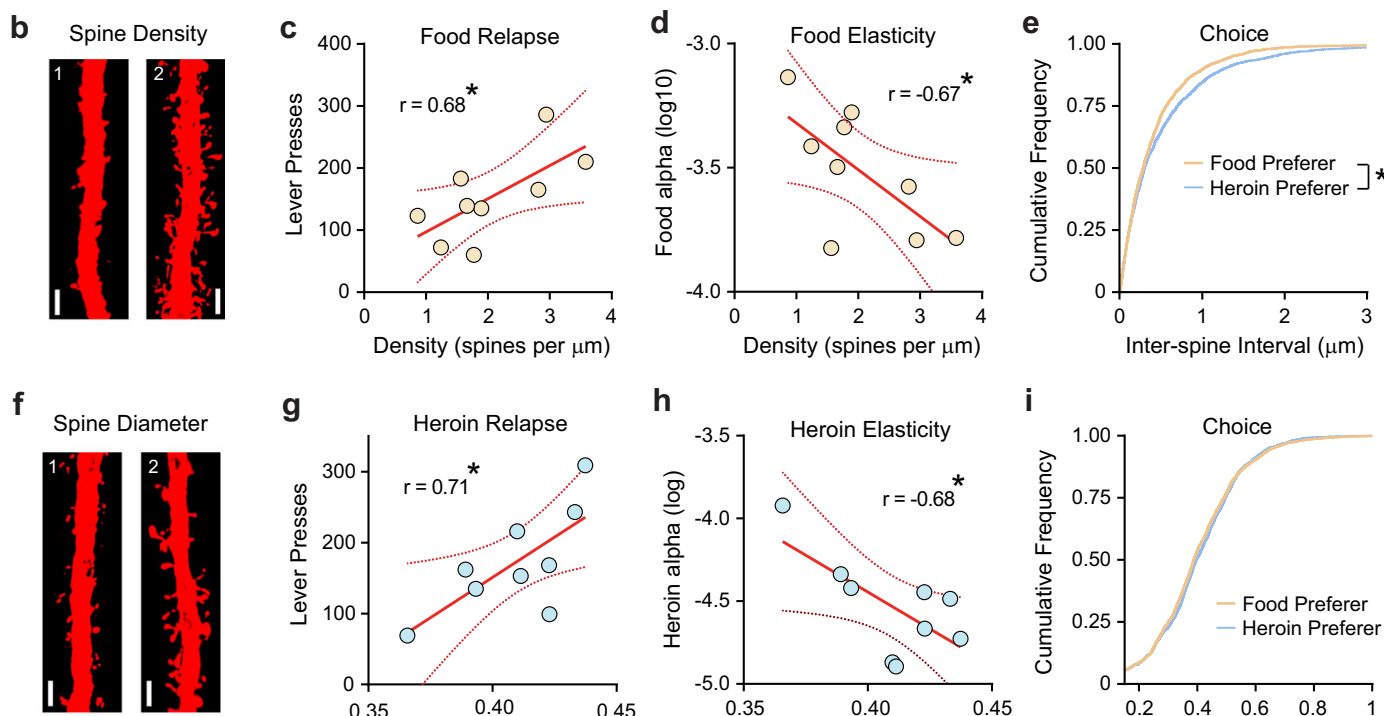

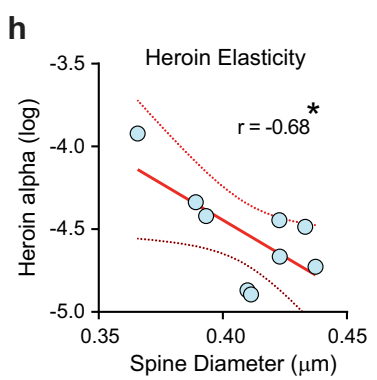

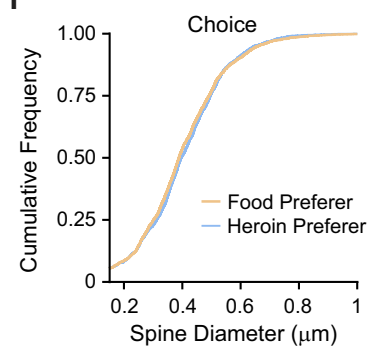

Fig. 6 IL $\rightarrow$ NAshell spines encode heroin choice and relapse. a Representative micrograph showing mCherry expression in an IL $\rightarrow$ NAshell neuron (scale bar $=20 \mu \mathrm{m}$ ). b Representative segments with (left) lowest and (right) highest spine density (scale bar $=3 \mu \mathrm{m}$ ). Data were obtained from four dendritic segments in nine rats. c IL-NAshell spine density positively correlates with food relapse (Pearson's $r=0.68, p=0.04$ ) and $\mathbf{d}$ negatively correlates with food alpha (Pearson's $r=-0.67, p=0.04$ ). e Cumulative frequency distribution showing lower inter-spine interval is associated with food preference (two-tailed two-sample Kolmogorov-Smirnov test, KS2 $=0.07, p=1.29 \times 10^{-5}$ ). f Representative segments showing (left) smallest and (right) largest spine head diameters from IL $\rightarrow$ NAshell neurons (scale bar $=3 \mu \mathrm{m}$ ). Data were obtained from four dendritic segments in nine rats. $\mathbf{g}$ Spine head diameter positively correlates with heroin relapse (Pearson's $r=0.71, p=0.03$ ) and $\mathbf{h}$ negatively correlates with heroin alpha (Pearson's $r=-0.68, p=0.04)$. $\mathbf{i}$ spine head diameter does not differ between heroin and food preferring rats. Total $n=9$ rats (food preferer $n=4$, heroin preferer $n=5$ ). ${ }^{\star} p<$ 0.05 significant correlation ( $\mathbf{c}, \mathbf{d}, \mathbf{g}, \mathbf{h}$ ) or comparing between food and heroin preference (e). 
between food and heroin) regardless of whether we used a behavioral economics model where animals had an extensive opioid self-administration history versus an abbreviated choice model with a more limited history of opioid exposure. This affords us a unique opportunity to examine neurobiological differences in these subpopulations going forward. Future studies should examine how the IL $\rightarrow$ NAshell pathway fits within a broader circuitry controlling choice between opioid and natural reward, and further probe the circumstances under which this pathway is capable of limiting opioid addiction-like behaviors.

\section{Methods}

Animals. All animal procedures followed guidelines approved by the University of Colorado Denver, Anschutz Medical Campus Institutional Animal Care and Use Committee. Subjects were age-matched (P55-60) male and female Wistar rats (Charles River, Raleigh, NC). Animals were single-housed in a temperature and humidity-controlled environment (lights on 7 a.m.-7 p.m.) with free access to standard laboratory chow and water. Our procedures followed the guidelines outlined in the Guide for the Care and Use of Laboratory Animals ${ }^{58}$. Rats were excluded from the final dataset if catheter patency was lost, if they were identified as outliers during statistical analysis (see Statistical Analyses), if they failed to meet specified behavioral criteria, or for poor placement and/or virus expression (where relevant).

Surgical procedures. Rats were anesthetized with ketamine/xylazine $(80 / 7 \mathrm{mg} / \mathrm{kg})$ and implanted with an intravenous catheter in the right jugular vein and intracranial cannulas (Plastics One, Roanoke, VA, USA) ${ }^{9,59}$ using the following coordinates relative to bregma: NAshell $+1.4 \mathrm{~mm}$ anterior-posterior $(\mathrm{A} / \mathrm{P}), \pm 0.8 \mathrm{~mm}$ mediolateral $(\mathrm{M} / \mathrm{L})$, and $-6.0 \mathrm{~mm}$ dorsoventral $(\mathrm{D} / \mathrm{V}) ; \mathrm{IL}+2.9 \mathrm{~mm} \mathrm{~A} / \mathrm{P}, \pm 0.6 \mathrm{~mm}$ $\mathrm{M} / \mathrm{L}$, and $-2.5 \mathrm{~mm} \mathrm{D} / \mathrm{V}$. Carprofen $(5 \mathrm{mg} / \mathrm{kg})$ and cefazolin $(30 \mathrm{mg} / \mathrm{kg}$ ) were administered to alleviate surgical pain and prevent infection. Rats were allowed to recover from surgery at least 1 week prior to virus infusions.

Chemogenetics approach. Under light isoflurane anesthesia, rats received bilateral injections of the virus into IL and NAshell (volume: $0.5 \mu \mathrm{l} / \mathrm{side}$, rate: $0.1 \mu \mathrm{l} / \mathrm{min}$ ). Injectors extended $3 \mathrm{~mm}$ beyond cannula for IL and $2 \mathrm{~mm}$ for NAshell. The NAshell was injected with a retrogradely transported AAV vector carrying Cre recombinase (AAVrg-Ef1 $\alpha$-Cre; $n=30$; Salk Institute; titer $1.1 \times 10^{12} \mathrm{GC} / \mathrm{ml}$ ). The IL was injected with viral vectors carrying inhibitory or excitatory designer receptors exclusively activated by designer drugs (Gi- or Gq-DREADDs; AAV2hSyn-DIO-hM4Di-mCherry; $n=15$; Addgene \#44362-AAV2; titer $3.5 \times 10^{12} \mathrm{GC} /$ $\mathrm{ml}$ or AAV2-hSyn-DIO-hM3Dq-mCherry; $n=15$; Addgene \#44361-AAV2; titer $3.5 \times 10^{12} \mathrm{GC} / \mathrm{ml}$ ). The DREADD ligand JHU37160 dihydrochloride (J60, HelloBio \#HB6261), dissolved in sterile water, was administered at a dose of $0.1 \mathrm{mg} / \mathrm{kg}$ $\left(1 \mathrm{ml} / \mathrm{kg}\right.$, IP) $15 \mathrm{~min}$ prior to testing ${ }^{60}$

Intracranial muscimol microinjections. Rats received bilateral microinjections of muscimol (MUS; volume: $0.3 \mu \mathrm{l} / \mathrm{side}$, rate: $0.25 \mu \mathrm{l} / \mathrm{min}$ ) or vehicle (sterile PBS) into the IL cortex through their intracranial cannula just prior to testing. Injectors extended $3 \mathrm{~mm}$ beyond the cannula. The dose of MUS ( $15 \mathrm{ng} / \mathrm{side}$ ) was based on previous work indicating this dose does not produce locomotor effects ${ }^{6}{ }^{6}$. The order of MUS versus vehicle alternated between within-subjects tests. At the end of the experiment, rats were microinjected with an equimolar, equi-volume fluorescenttagged MUS (MUS-TMR-X) prior to histological procedures in order to visualize the spread of MUS-TMR-X at the injection site ${ }^{62}$.

Food self-administration for behavioral economics. Food self-administration training $(1 \mathrm{~h} /$ session/weekday) occurred in standard operant chambers inside ventilated, sound-attenuating cubicles (Med Associates, St. Albans, VT, USA). Rats were trained to self-administer food pellets (Bio-Serv, \#F0165, $45 \mathrm{mg}$ ) on a fixedratio 1 (FR1) schedule of reinforcement. During food self-administration $(1 \mathrm{~h} /$ session/weekday), only the right retractable lever was extended. Three pellets and a $3.5 \mathrm{kHz}$ tone cue $(5 \mathrm{sec})$ were delivered each time the FR requirement was reached, and the lever retracted during cue presentation. After five sessions, training progressed through five additional FR schedules (FR3, FR5, FR10, FR32, and FR100) with at least 2 days on each FR, or until $\leq 20 \%$ variability in rewards earned was achieved.

Heroin self-administration for behavioral economics. After completing food self-administration, rats began daily heroin self-administration training (FR1; $3 \mathrm{~h} /$ session/weekday), during which only the left lever was extended. Heroin (diamorphine $\mathrm{HCl}$; NIDA Drug Supply Program) was dissolved in $0.9 \%$ sterile saline. After an FR requirement was reached, a heroin infusion $(0.04 \mathrm{mg} / 50 \mu \mathrm{l} / 2.85 \mathrm{sec})$ was delivered, a light above the left lever was turned on $(5 \mathrm{sec})$, and the lever retracted during cue presentation. After at least five sessions, animals progressed over additional FR schedules (FR3, FR10, FR21, FR32, FR45, FR100, FR316,
FR1000), with at least 2 days on each FR, or until $\leq 20 \%$ variability in rewards earned was achieved. To prevent infection and catheter occlusion, cefazolin and taurolidine-citrate solution were administered after each self-administration session

Generating demand curves for behavioral economics. We used a betweensession behavioral economics paradigm where male rats were allowed to selfadminister food or heroin in daily sessions of fixed duration ${ }^{28}$. Food and heroin demand curves were generated separately for each animal in Matlab (Mathworks, $\mathrm{R} 2019 \mathrm{~b}$ ), using the average responding over the last two sessions on each FR, according to the formula ${ }^{63}$,

$$
\ln Q=\ln Q_{0}+k\left(e^{-\alpha Q_{0} C}-1\right)
$$

$\mathrm{C}$ indicates consumption (i.e., number of pellets earned or heroin infusions received at each price-point, see ref. ${ }^{38}$ :) and constant $\mathrm{k}$ (set to 8.85) specifies the range of consumption values. From the resulting demand curves, we extracted the following variables: $\alpha$ (demand elasticity; rate at which consumption declines with increasing price), $\mathrm{Q}_{0}$ (consumption at null cost; intrinsic motivational efficacy of the reward), Pmax (maximum price paid before demand rapidly declines; derived using a solver algorithm), and Omax (the maximum rate of responding to defend desired consumption $)^{28,63,64}$. We also calculated EV according to the formula ${ }^{25}$ :

$$
\mathrm{EV}=\left(100 \cdot \alpha \cdot k^{1.5}\right)^{-1}
$$

Because EV is independent of reinforcer magnitude ${ }^{25}$, it is useful for comparing different reinforcers directly. We also compared normalized Pmax values between food and heroin demand by dividing Pmax by $\mathrm{Q}_{0}$ values, as described previously ${ }^{64}$

Choice procedures after behavioral economics. Choice procedures were implemented at the end of heroin self-administration in the behavioral economics study. During this phase, rats were given 14 trials to choose food (three pellets + food-tone) or heroin (0.04 mg infusion + heroin-light). During each trial, both right and left levers were extended, and the rat needed to reach an FR3 requirement on one of the levers to earn a food or heroin reward. Afterward, both levers retracted for a $10 \mathrm{~min}$ time-out between trials (house-light off and levers retracted). The first five-choice sessions contained two forced-choice trials for each reinforcer (four total trials) during which only one lever was extended and ten free-choice trials with two levers. Forced choice trials were administered in the order: foodheroin-food-heroin. Afterward, rats moved to free-choice sessions (both levers extended for all 14 trials) for the remainder of choice training. Following at least three free-choice sessions or until there was $\leq 20 \%$ variability in total choices over the last two sessions, rats started choice tests, separated by at least two stable choice sessions. Prior to each test, rats were injected with J60 or vehicle in a randomized, counterbalanced order. The choice was expressed as the ratio of heroin choices over total choices.

Abbreviated self-administration choice protocol. For the IL-MUS experiment, we used a protocol wherein male and female rats were trained to self-administer food and heroin simultaneously during daily self-administration sessions. The parameters were equivalent to those described above for the behavioral economics experiment, except that sessions were a total of $3 \mathrm{~h}$ long, and the number of selfadministration days was held constant across all rats. After six sessions on an FR1 schedule of reinforcement, rats progressed to an FR3 scheduled for three sessions and then proceeded to free-choice sessions thereafter. These free-choice sessions were identical to those used after behavioral economics procedures except the session duration was held constant at $3 \mathrm{~h}$ (e.g., not capped at 14 trials). After at least nine sessions on choice, and meeting stability criteria $(\leq 20 \%$ variability in total choices over the last two sessions), rats began testing after intra-IL microinfusion of the vehicle or MUS (see Intracranial muscimol microinfusions). Rats underwent two choice sessions in between test days using a within-subject design with treatment order counterbalanced across subjects.

Cued relapse testing. After rats completed choice testing, they were returned to self-administration procedures to ensure recent exposure to both heroin and food (and their respective cues) across all rats (including heroin and food preferers). After three FR3 reacquisition sessions, rats began cued relapse testing. Thus, the relapse terminology used throughout this manuscript refers specifically to relapse during acute withdrawal (except Supplementary Fig. 5). For the behavioral economics study, each lever was extended separately in two 30-min bins, separated by a $10 \mathrm{~min}$ time-out (levers retracted, house-light off). Pressing on each lever resulted in cue delivery (food-tone or heroin-light) on an FR3 schedule. Levers retracted during cue delivery, as during self-administration sessions. The order in which the food or heroin lever was presented was counterbalanced across rats and tests. For the IL-MUS experiment, the relapse test was conducted with both levers (food and heroin) available simultaneously for the 1-h test session. Each rat received drug (J60 or IL-MUS) or vehicle treatments in a randomized, counterbalanced order separated by at least two FR3 reacquisition sessions.

Histology and immunohistochemistry. Rats were transcardially perfused with $0.9 \%$ saline followed by $10 \%$ formalin. Brains were extracted, post-fixed in 
formalin, cryoprotected in $20 \%$ sucrose, and sectioned $(40 \mu \mathrm{m})$ on a cryostat (Leica, CM-1950). Free-floating sections were blocked in $2 \%$ normal donkey serum (NDS) in phosphate-buffered saline with $0.3 \%$ Triton X-100 (PBS-T) and incubated overnight at $4{ }^{\circ} \mathrm{C}$ with rabbit anti-cFos (1:2000, Millipore Cat\# ABE457, RRID: AB_2631318) and/or chicken anti-mCherry (1:5000, LifeSpan, Cat\# LS-C204825, RRID: AB_2716246). Sections were then rinsed in PBS-T and incubated for $2 \mathrm{~h}$ in PBS-T with $2 \%$ of NDS containing fluorescent Alexa-conjugated secondary antibodies (1:500, Jackson ImmunoResearch Labs). NeuroTrace fluorescent Nissl 640/660 (1:500, Thermo Fisher Scientific, RRID:AB_2572212) was applied during the secondary antibody step, or DAPI coverslipping media was used as a counterstain. Images were acquired with an Olympus slide scanner microscope (VS120; $2 \mathrm{x}$ or 10x air objective; Olympus, Center Valley, PA). Ten rats were excluded from chemogenetics experiments based on low or misplaced virus expression.

Cell counts. The chemogenetic approach was validated using Fos as a measure of neuronal activity after $\mathrm{J} 60$ or vehicle administration in male and female rats. The Gi (hM4Di)- or Gq (hM3Dq)- DREADD was restricted to the IL-NAshell pathway using the combinatorial viral strategy described above (see Chemogenetics approach). To verify Gi-DREADD functionality, rats received an injection of cocaine (cocaine hydrochloride, $10 \mathrm{mg} / \mathrm{kg}, 1 \mathrm{ml} / \mathrm{kg}$ IP, NIDA Drug Supply) to induce Fos, $15 \mathrm{~min}$ after $\mathrm{J} 60(0.1 \mathrm{mg} / \mathrm{kg}, 1 \mathrm{ml} / \mathrm{kg})$ or sterile water pretreatment. To verify Gq-DREADD functionality, rats received J60 $(0.1 \mathrm{mg} / \mathrm{kg}, 1 \mathrm{ml} / \mathrm{kg} \mathrm{IP})$ or vehicle (sterile water). All injections were given in the home cage, and all rats were perfused transcardially $2 \mathrm{~h}$ after J60 or vehicle. Histology and immunohistochemistry were performed as described above, and images were analyzed using Imaris software (Bitplane). A region of interest (ROI) was set and the number of Fos+, hM4Di-, or hM3Dq-mCherry+ neurons in the IL was automatically quantified using the Imaris spot detection function on thresholded images. The number of co-expressing neurons in each section were determined using the colocalize spot function. For all rats, the number of cells was averaged within each hemisphere across at least three sections.

Dendritic spine analyses. Spine analyses were performed in DREADD-infected, mCherry-tagged neurons from the behavioral economics study ${ }^{65}$. Dendritic segments from IL $\rightarrow$ NAshell neurons selected for spine analysis were visually connected to the soma. Z-series were acquired with a confocal microscope (Olympus FV1200) using a 60x oil immersion objective (1.4 NA), at $1024 \times 512$ frame size using $4.1 \mathrm{x}$ digital zoom and a $0.1 \mu \mathrm{m}$ step size. mCherry was excited using a $488 \mathrm{~nm}$ argon-ion laser line and overall, imaging parameters were held constant during the acquisition process. Apical dendrite segments from layer $5 / 6$ pyramidal neurons were imaged in layer $2 / 3(\sim 300 \mu \mathrm{m}$ from the soma $), \sim 10 \mu \mathrm{m}$ before branching into the apical tuft. Images were cropped and deconvolved using Huygens (SVI) software. Dendritic segments (mean \pm SEM length: $65.6 \pm 0.38 \mu \mathrm{m}$ ) were imported into Imaris (Bitplane) software and spines were semiautomatically drawn using the filament module. Spine density and head diameter values were determined from four dendritic segments per animal.

Statistical analyses. All statistical tests were performed in Prism (GraphPad, 8.0) and Matlab (Mathworks, 2019b). Choice and relapse tests were analyzed using repeated measures analysis of variance (ANOVA), followed by Bonferroni corrected planned comparisons, or by using two-tailed, planned comparison paired $t$ tests. Cell counts for Fos expression analyses were analyzed using two-tailed unpaired $t$-tests. Linear relationships between variables were analyzed using Pearson's Correlation Coefficient. Principal component analysis was conducted using $\mathrm{z}$-transform scaled data in Matlab. Cumulative frequency distributions were compared using two-sample Kolmogorov-Smirnov tests in Matlab. Outliers were identified using ROUT analysis. Significance was defined as alpha $<0.05$ and data were graphed as mean \pm standard error of the mean (SEM).

Reporting Summary. Further information on research design is available in the Nature Research Reporting Summary linked to this article.

\section{Data availability}

The datasets generated during and/or analysed during the current study are available from the corresponding author on reasonable request. Source data are provided with this paper.

Received: 24 July 2020; Accepted: 19 July 2021;

Published online: 09 August 2021

\section{References}

1. NSDUH. in Substance Abuse and Mental Health Services Administration. Key substance use and mental health indicators in the United States: Results from the 2018 National Survey on Drug Use and Health (HHS Publication No. PEP19-5068). Rockville, MD: Center for Behavioral Health Statistics and
Quality, Substance Abuse and Mental Health Services Administration (2019). Retrieved from https://www.samhsa.gov/data/.

2. Moorman, D. E. et al. Differential roles of medial prefrontal subregions in the regulation of drug seeking. Brain Res. 1628, 130-146 (2015).

3. Peters, J., Kalivas, P. W. \& Quirk, G. J. Extinction circuits for fear and addiction overlap in prefrontal cortex. Learn Mem. 16, 279-288 (2009).

4. Kalivas, P. W. The glutamate homeostasis hypothesis of addiction. Nat. Rev. Neurosci. 10, 561-572 (2009).

5. Bossert, J. M. et al. Ventral medial prefrontal cortex neuronal ensembles mediate context-induced relapse to heroin. Nat. Neurosci. 14, 420-422 (2011).

6. Bossert, J. M. et al. Role of projections from ventral medial prefrontal cortex to nucleus accumbens shell in context-induced reinstatement of heroin seeking. J. Neurosci. 32, 4982-4991 (2012).

7. Rogers, J. L., Ghee, S. \& See, R. E. The neural circuitry underlying reinstatement of heroin-seeking behavior in an animal model of relapse. Neuroscience 151, 579-588 (2007).

8. Peters, J., LaLumiere, R. T. \& Kalivas, P. W. Infralimbic prefrontal cortex is responsible for inhibiting cocaine seeking in extinguished rats. J. Neurosci. 28, 6046-6053 (2008)

9. Augur, I. F. et al. Chemogenetic activation of an extinction neural circuit reduces cue-induced reinstatement of cocaine seeking. J. Neurosci. 36, 10174-10180 (2016).

10. Cameron, C. M. et al. Increased cocaine motivation is associated with degraded spatial and temporal representations in IL-NAc neurons. Neuron 103, 80-91 e7 (2019).

11. Ma, Y. Y. et al. Bidirectional modulation of incubation of cocaine craving by silent synapse-based remodeling of prefrontal cortex to accumbens projections. Neuron 83, 1453-1467 (2014).

12. Halladay, L. R. et al. Prefrontal regulation of punished ethanol selfadministration. Biol. Psychiatry 87, 967-978 (2020).

13. Higgins, S. T., Heil, S. H. \& Lussier, J. P. Clinical implications of reinforcement as a determinant of substance use disorders. Annu. Rev. Psychol. 55, 431-461 (2004).

14. Ahmed, S. H., Lenoir, M. \& Guillem, K. Neurobiology of addiction versus drug use driven by lack of choice. Curr. Opin. Neurobiol. 23, 581-587 (2013).

15. Cantin, L. et al. Cocaine is low on the value ladder of rats: possible evidence for resilience to addiction. PLoS ONE 5, el1592 (2010).

16. Caprioli, D. et al. Persistent palatable food preference in rats with a history of limited and extended access to methamphetamine self-administration. Addict. Biol. 20, 913-926 (2015).

17. Lenoir, M. et al. Intense sweetness surpasses cocaine reward. PLoS ONE 2 , e698 (2007).

18. Venniro, M. et al. Incubation of methamphetamine but not heroin craving after voluntary abstinence in male and female rats. Neuropsychopharmacology 42, 1126-1135 (2017).

19. Madsen, H. B. \& Ahmed, S. H. Drug versus sweet reward: greater attraction to and preference for sweet versus drug cues. Addict. Biol. 20, 433-444 (2015).

20. Venniro, M., Caprioli, D. \& Shaham, Y. Animal models of drug relapse and craving: from drug priming-induced reinstatement to incubation of craving after voluntary abstinence. Prog. Brain Res. 224, 25-52 (2016).

21. Reiner, D. J. et al. Role of projections between piriform cortex and orbitofrontal cortex in relapse to fentanyl seeking after palatable food choiceinduced voluntary abstinence. J. Neurosci. 40, 2485-2497 (2020).

22. Venniro, $M$. et al. The anterior insular cortex $\rightarrow>$ central amygdala glutamatergic pathway is critical to relapse after contingency management. Neuron 96, 414-427 e8 (2017).

23. Anthony, J. C., Warner, L. A. \& Kessler, R. C. in Addictive behaviors: Readings on etiology, prevention, and treatment (eds. Marlatt, G. A. \& VandenBos, G. R.) Ch.1 (American Psychological Association, 1997).

24. Anthony, J. C. \& Petronis, K. R. Cocaine and heroin dependence compared: evidence from an epidemiologic field survey. Am. J. Public Health 79, 1409-1410 (1989).

25. Hursch, S.R. in The Wiley Blackwell Handbook of Operant and Classical Conditioning (eds McSweeney, F. K. \& Murphy, E. S.) Ch. 12 (Wiley, 2014).

26. Mohammadkhani, A. et al. Orexin-1 receptor signaling in ventral pallidum regulates motivation for the opioid remifentanil. J. Neurosci. 39, 9831-9840 (2019).

27. Fragale, J. E. et al. The role of orexin-1 receptor signaling in demand for the opioid fentanyl. Neuropsychopharmacology 44, 1690-1697 (2019).

28. Bentzley, B. S., Jhou, T. C. \& Aston-Jones, G. Economic demand predicts addiction-like behavior and therapeutic efficacy of oxytocin in the rat. Proc. Natl Acad. Sci. USA 111, 11822-11827 (2014).

29. Schwartz, L. P. et al. Heroin and saccharin demand and preference in rats. Drug Alcohol Depend. 178, 87-93 (2017).

30. van Duuren, E. et al. Pharmacological manipulation of neuronal ensemble activity by reverse microdialysis in freely moving rats: a comparative study of the effects of tetrodotoxin, lidocaine, and muscimol. J. Pharm. Exp. Ther. 323 61-69 (2007) 
31. Armbruster, B. N. et al. Evolving the lock to fit the key to create a family of G protein-coupled receptors potently activated by an inert ligand. Proc. Natl Acad. Sci. USA 104, 5163-5168 (2007).

32. Alexander, G. M. et al. Remote control of neuronal activity in transgenic mice expressing evolved G protein-coupled receptors. Neuron 63, 27-39 (2009).

33. Warren, B. L. et al. Separate vmPFC ensembles control cocaine selfadministration versus extinction in rats. J. Neurosci. 39, 7394-7407 (2019).

34. MacKillop, J. \& Murphy, J. G. A behavioral economic measure of demand for alcohol predicts brief intervention outcomes. Drug Alcohol Depend. 89, 227-233 (2007).

35. Walsh, S. L. et al. Cocaine abuse versus cocaine dependence: cocaine selfadministration and pharmacodynamic response in the human laboratory. Drug Alcohol Depend. 106, 28-37 (2010).

36. Greenwald, M. K. Effects of experimental unemployment, employment and punishment analogs on opioid seeking and consumption in heroin-dependent volunteers. Drug Alcohol Depend. 111, 64-73 (2010).

37. Ahmed, S. H. Validation crisis in animal models of drug addiction: beyond non-disordered drug use toward drug addiction. Neurosci. Biobehav. Rev. 35, 172-184 (2010).

38. James, M. H. et al. Increased number and activity of a lateral subpopulation of hypothalamic orexin/hypocretin neurons underlies the expression of an addicted state in rats. Biol. Psychiatry 85, 925-935 (2019).

39. Fattore, L. \& Diana, M. Drug addiction: an affective-cognitive disorder in need of a cure. Neurosci. Biobehav Rev. 65, 341-361 (2016).

40. Kalivas, P. W. \& Volkow, N. D. The neural basis of addiction: a pathology of motivation and choice. Am. J. Psychiatry 162, 1403-1413 (2005).

41. Pfarr, S. et al. Losing Control: excessive alcohol seeking after selective inactivation of cue-responsive neurons in the infralimbic cortex. J. Neurosci. 35, 10750-10761 (2015).

42. Laque, A. et al. Anti-relapse neurons in the infralimbic cortex of rats drive relapse-suppression by drug omission cues. Nat. Commun. 10, 3934 (2019).

43. Pfarr, S. et al. Choice for drug or natural reward engages largely overlapping neuronal ensembles in the infralimbic prefrontal cortex. J. Neurosci. 38, 3507-3519 (2018).

44. Barreiros, I. V., Panayi, M. C. \& Walton, M. E. Organization of afferents along the anterior-posterior and medial-lateral axes of the rat orbitofrontal cortex. Neuroscience 460, 53-68 (2021).

45. Guillem, K. et al. Neuronal representation of individual heroin choices in the orbitofrontal cortex. Addict. Biol. 23, 880-888 (2018).

46. Augier, E. et al. A molecular mechanism for choosing alcohol over an alternative reward. Science 360, 1321-1326 (2018).

47. Parkes, S. L., Bradfield, L. A. \& Balleine, B. W. Interaction of insular cortex and ventral striatum mediates the effect of incentive memory on choice between goal-directed actions. J. Neurosci. 35, 6464-6471 (2015).

48. Pecina, S. \& Berridge, K. C. Hedonic hot spot in nucleus accumbens shell: where do mu-opioids cause increased hedonic impact of sweetness? J. Neurosci. 25, 11777-11786 (2005).

49. Johnson, P. I. et al. Reward shifts and motor responses following microinjections of opiate-specific agonists into either the core or shell of the nucleus accumbens. Psychopharmacol. 120, 195-202 (1995).

50. McBride, W. J., Murphy, J. M. \& Ikemoto, S. Localization of brain reinforcement mechanisms: intracranial self-administration and intracranial place-conditioning studies. Behav. Brain Res. 101, 129-152 (1999).

51. Baldo, B. A. \& Kelley, A. E. Discrete neurochemical coding of distinguishable motivational processes: insights from nucleus accumbens control of feeding. Psychopharmacol. 191, 439-459 (2007).

52. Hanlon, E. C. et al. Increases in food intake or food-seeking behavior induced by GABAergic, opioid, or dopaminergic stimulation of the nucleus accumbens: is it hunger? Psychopharmacol. 172, 241-247 (2004).

53. Venniro, M., Caprioli, D. \& Shaham, Y. Novel models of drug relapse and craving after voluntary abstinence. Neuropsychopharmacology 44, 234-235 (2019).

54. Canchy, L. et al. Pharmacokinetics trumps pharmacodynamics during cocaine choice: a reconciliation with the dopamine hypothesis of addiction. Neuropsychopharmacology 46, 288-296 (2021).

55. Lenoir, M. et al. Extended heroin access increases heroin choices over a potent nondrug alternative. Neuropsychopharmacology 38, 1209-1220 (2013).

56. Lenoir, M. \& Ahmed, S. H. Supply of a nondrug substitute reduces escalated heroin consumption. Neuropsychopharmacology 33, 2272-2282 (2008).

57. McConnell, S. A. et al. Demand for fentanyl becomes inelastic following extended access to fentanyl vapor self-administration. Neuropharmacology 182, 108355 (2021).
58. National Research Council., Committee for the Update of the Guide for the Care and Use of Laboratory Animals., Institute for Laboratory Animal Research. \& Division of Earth and Life Studies. Guide for the Care and Use of Laboratory Animals (National Academies Press, 2011).

59. Vazquez, M. et al. Acute ovarian hormone treatment in freely cycling female rats regulates distinct aspects of heroin seeking. Learn Mem. 27, 6-11 (2020).

60. Bonaventura, J. et al. High-potency ligands for DREADD imaging and activation in rodents and monkeys. Nat. Commun. 10, 4627 (2019).

61. Sierra-Mercado, D., Padilla-Coreano, N. \& Quirk, G. J. Dissociable roles of prelimbic and infralimbic cortices, ventral hippocampus, and basolateral amygdala in the expression and extinction of conditioned fear. Neuropsychopharmacology 36, 529-538 (2010).

62. Misane, I. et al. GABA(A) receptor activation in the CA1 area of the dorsal hippocampus impairs consolidation of conditioned contextual fear in C57BL/6J mice. Behav. Brain Res. 238, 160-169 (2013).

63. Hursh, S. R. \& Silberberg, A. Economic demand and essential value. Psychol. Rev. 115, 186-198 (2008).

64. Bentzley, B. S., Fender, K. M. \& Aston-Jones, G. The behavioral economics of drug self-administration: a review and new analytical approach for withinsession procedures. Psychopharmacol. 226, 113-125 (2013).

65. Siemsen, B. M. et al. Biphasic effect of abstinence duration following cocaine self-administration on spine morphology and plasticity-related proteins in prelimbic cortical neurons projecting to the nucleus accumbens core. Brain Struct. Funct. 224, 741-758 (2019).

\section{Acknowledgements}

The authors would like to thank the NIDA Drug Supply Program for supplying heroin and cocaine, and Dr. Michael Scofield for helpful advice with dendritic spine analyses. This research was funded by NIDA R01 DA045836, R21 DA044524, K01 DA038235 (J.P.), DP5 OD026407 (J.A.H.), and K99 DA045765 (M.H.J.)

\section{Author contributions}

J.A.H., G.A.-J., M.H.J., and J.P. designed the study. J.A.H., G.G., M.R.M., M.J., and J.P performed experiments. J.A.H., G.G., and J.P. analyzed data. J.A.H. and J.P. wrote the manuscript with input from all authors.

\section{Competing interests}

The authors declare no competing interests.

\section{Additional information}

Supplementary information The online version contains supplementary material available at https://doi.org/10.1038/s41467-021-25080-x.

Correspondence and requests for materials should be addressed to J.P.

Peer review information Nature Communications thanks Wolfgang Sommer and the other, anonymous, reviewer(s) for their contribution to the peer review of this work.

Reprints and permission information is available at http://www.nature.com/reprints

Publisher's note Springer Nature remains neutral with regard to jurisdictional claims in published maps and institutional affiliations.

Open Access This article is licensed under a Creative Commons Attribution 4.0 International License, which permits use, sharing, adaptation, distribution and reproduction in any medium or format, as long as you give appropriate credit to the original author(s) and the source, provide a link to the Creative Commons license, and indicate if changes were made. The images or other third party material in this article are included in the article's Creative Commons license, unless indicated otherwise in a credit line to the material. If material is not included in the article's Creative Commons license and your intended use is not permitted by statutory regulation or exceeds the permitted use, you will need to obtain permission directly from the copyright holder. To view a copy of this license, visit http://creativecommons.org/ licenses/by/4.0/.

(C) The Author(s) 2021 